doi: $10.1016 /$ j.jinf.2005.10.020

Copyright (c) 2005 The British Infection Society Published by Elsevier Ltd.

\title{
The incidence of Bordetella pertussis infections estimated in the population from a combination of serological surveys
}

\section{Hester E. de Melker ${ }^{a}$, Florens G.A. Versteegh ${ }^{\text {b, }} *$, Joop F.P. Schellekens ${ }^{c}$, Peter F.M. Teunis ${ }^{d}$ and Mirjam Kretzschmar ${ }^{a}$}

${ }^{a}$ Department of Infectious Diseases Epidemiology, National Institute of Public Health and the Environment, Bilthoven, The Netherlands

${ }^{b}$ Department of Pediatrics, Groene Hart Hospital, P.O. Box 1098, 2800 BB Gouda, The Netherlands

${ }^{\mathrm{C} D}$ Diagnostic Laboratory of Infectious Diseases and Perinatal Screening, National Institute of Public Health and the Environment, Bilthoven, The Netherlands ${ }^{\mathrm{d} C}$ Computerization and Methodological Consultancy Unit, National Institute of Public Health and the Environment, Bilthoven, The Netherlands

* Corresponding author. Tel.: +31 182 505050; fax: +31 182505700.

\section{Summary}

\section{Objectives}

Bordetella pertussis circulates even in highly vaccinated populations. There is a considerable amount of infection in adults. For designing more effective vaccination schedules it is important to quantify the age-dependent relation between the number of notified cases and the number of infections.

\section{Methods}

We used a statistical relationship between the time since infection and the IgG antibody titers against pertussis toxin, derived from a longitudinal data set, to estimate time since infection for all individuals in a cross-sectional populationbased study (1995-1996) based on their titers. Age-specific incidence of infection with $B$. pertussis was calculated and compared with the age-distribution of notified cases of pertussis in 1994-1996.

\section{Results}

Estimated incidence of infection was $6.6 \%$ per year for 3-79-year olds, annual incidence of notified cases $0.01 \%$. Estimated age-specific incidence of infection was lowest for 3-4-year olds (3.3\%) and increased gradually up to the age of 2024 years $(10.8 \%)$. The number of notified cases was highest for 3-9-year olds.

\section{Conclusions}

In the Dutch population $B$. pertussis infections occur more frequently and in elder age-categories then suggested by notifications. Mathematical modeling could explore what booster vaccination strategies are most effective in reducing severe disease among not (completely) vaccinated infants. 


\section{Introduction}

Despite widespread vaccination, infection with Bordetella pertussis remains a cause of considerable morbidity even in countries with high vaccination coverage. $1,2,3,4$ and 5 The continuing circulation of the pathogen is attributed to waning of vaccine-induced immunity, which leads to the occurrence of (often undiagnosed) pertussis infection among previously vaccinated children, adolescents and adults. $6,7,8,9,10,11,12$ and 13 Like others, we have shown that the definition of reliable criteria for positivity associated with high levels of IgG to pertussis toxin (IgG-PT) in a single serum sample (one-point serology) can greatly enhance the detection of pertussis infections. ${ }^{4,7}$ and 10

Inclusion of positive one-point serology as laboratory confirmation for notification in the Netherlands has increased the notification rate considerably, especially among older children and adults. ${ }^{1}$ However, the use of surveillance data of notified infections for understanding the epidemiology of pertussis remains limited by the lack of registration or notification discipline as well as age-dependent diagnosis and reporting. Circulation of $B$. pertussis in vaccinated children, adolescents and adults plays an important role in the continuing transmission of the pathogen to infants too young to be vaccinated, in whom disease is most severe and possibly fatal. $8,11,12,13,14,15,16,17,18,19$ and 20

To design better preventive measures, for example by determining the optimal ages for booster vaccinations, insight is needed into the age-specific incidence of all infections with $B$. pertussis as opposed to only those symptomatic infections that are diagnosed and reported. Information about the seroprevalence of IgG-PT antibodies in the general population in combination with knowledge about the rate of decline of IgG-PT antibody levels after infection with $B$. pertussis offers the opportunity to study the incidence of infection in various age groups in the population irrespective of clinical course, diagnosis and reporting frequency. Pertussis toxin is expressed only by $B$. pertussis and cross-reacting antigens have not been described. ${ }^{21}$ and 22

Furthermore IgG-PT responses occur in most patients with $B$. pertussis infection and high levels persist only temporarily. ${ }^{10}$ We estimated the incidence of $B$. pertussis infections in the population using a novel two-stage approach. A statistical description of the decline in antibody levels after infection as derived from a small scale longitudinal study ${ }^{23}$ was combined with data of the age-specific distribution of IgG-PT in sera derived from a large scale cross-sectional study of the general population ${ }^{10}$ to estimate the age-specific incidences of infection for the age range 3-79 years. These were compared with notification data of reported clinical cases of pertussis. Implications for vaccination-strategy are discussed.

\section{Material and methods}

\section{Collection of sera from population and patients}

Detailed descriptions of the collection of sera from the general population in the Netherlands $(n=7756)$ and of follow-up sera from patients with diagnosed clinical pertussis $(n=85)$ have been published elsewhere. ${ }^{10,23}$ and 24 In short, for the cross-sectional study in the general population, 40 municipalities were sampled with probabilities proportional to their population size. An age-stratified sample (classes $0,1-4,5-9, \ldots 75-79$ years) of 380 individuals was randomly selected from each municipality. Subjects were asked to give a blood sample. Samples were collected in the period from October 1995 to December 1996 and 
stored at $-70{ }^{\circ} \mathrm{C}$ until use. The participation rate was $55 \%$. Sufficient serum for pertussis serology was available for 7756 of 8359 participants.

In a longitudinal study, ${ }^{23}$ for 86 episodes 313 follow-up samples from 85 patients clinically diagnosed with pertussis (paroxysmal cough lasting more than 2 weeks) were obtained from one pediatric practice in the period 1989-2000. One patient had a second episode of (clinically confirmed) pertussis during the follow-up period: both episodes have been included in this analysis. ${ }^{25}$ The follow-up period ranged from 6 months to 11 years and the number of serial sera per patient ranged from 2 to 11 . Eleven episodes were from patients less than 6 months of age at the time they contracted pertussis, 69 episodes were from patients between 6 months and 17 years of age, and six patients were between 30 and 41 years old.

\section{Notification data}

Pertussis notification data for the period 1994-1996 were obtained from the Dutch Inspectorate of Health. The case definition included clinical symptoms and laboratory confirmation (or close contact with a person with laboratory-confirmed pertussis). The clinical symptoms in the case definition are a serious cough, lasting more than 2 weeks, coughing attacks, or coughing followed by vomiting in combination with at least one of the following symptoms/findings: apnea, cyanosis, characteristic cough with whooping, subconjunctival bleeding, or leucocytosis.

Laboratory confirmation was defined as either a positive culture of $B$. pertussis or B. parapertussis, or positive two-point serology. Two-point serology was considered positive if a 4-fold rise of IgG-antibodies against pertussis toxin in paired sera was found to be a concentration of at least $20 \mathrm{U} / \mathrm{ml}$. Only since April 1997 , positivity of one-point serology has been formally included in the case definition as being acceptable as laboratory confirmation of pertussis for notification (criterion: IgG-PT concentration in single serum $>100 \mathrm{U} / \mathrm{ml}$ ). However, already in the years before 1997, a small fraction of the patients with positive one-point serology was reported. ${ }^{1}$

\section{Antibody assay}

In the longitudinal study, patient sera had been submitted immediately after sampling and were assayed in the routine setting of the serology laboratory of our institute within 4 days after receipt. In the cross-sectional study, sera that had been collected in 1995/1996 were assayed in 1997/1998 in the same routine setting at a rate of approximately 200 sera per week. The IgG-PT was measured by ELISA as previously described. ${ }^{26}$ The IgG-PT assay has an upper limit $(500 \mathrm{U} / \mathrm{ml})$ above which the values are not further differentiated. The lower detection limit of the assay is $5 \mathrm{U} / \mathrm{ml}$. Results are expressed in "local $\mathrm{U} / \mathrm{ml}$ ".

\section{Analysis}

In Teunis et al. ${ }^{23}$ a skewed hyperbolic function was used to describe the relationship between the log time since infection with $B$. pertussis and the log IgG-PT antibody titer. This four-parameter function was fitted to the data for each individual patient from the longitudinal study of diagnosed clinical pertussis patients yielding a set of response curves with variation among individuals (Fig. 1). On a linear time scale, the antibody response rises very fast (within a few days) and then declines very slowly over a period of several years. Therefore, the rising part of the response curve may safely be neglected and an inverse function 
can be determined on the basis of the long monotone declining part of the response. For every value of the log titer an average time since infection can be calculated (Fig. 1).

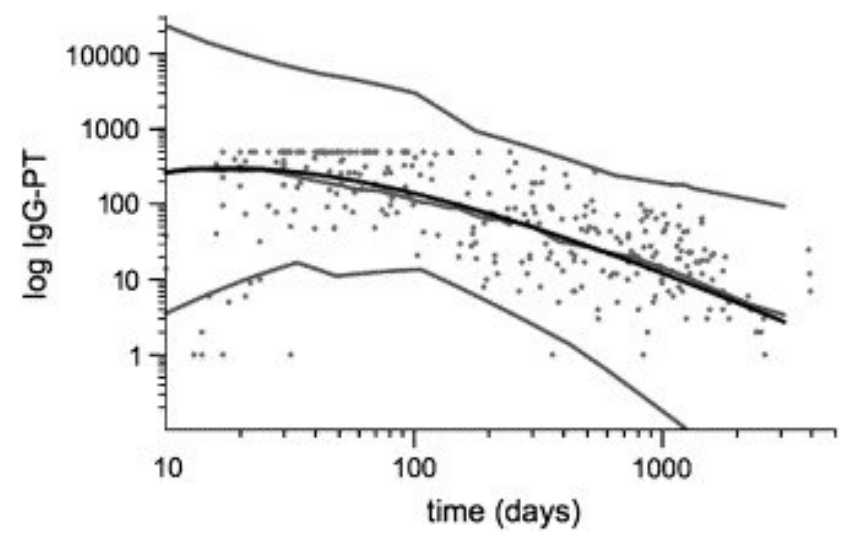

Figure 1. Measured IgG-PT concentration (titer) as a function of the time since last infection (for the study population in Teunis et al. ${ }^{23}$ ). The black line shows the point-wise average of individual estimates, the gray lines show the point wise $2.5 \%, 50 \%$ (median) and $95 \%$ percentiles illustrating the magnitude of individual variation in responses. The dots are individual results.

We used those point-wise averages of the inverse response curves to estimate the times since last infection for the cross-sectional study population. Fig. 2 shows the IgG-PT antibody distribution in the participants from the crosssectional population-based study (adapted from ${ }^{10}$ ).

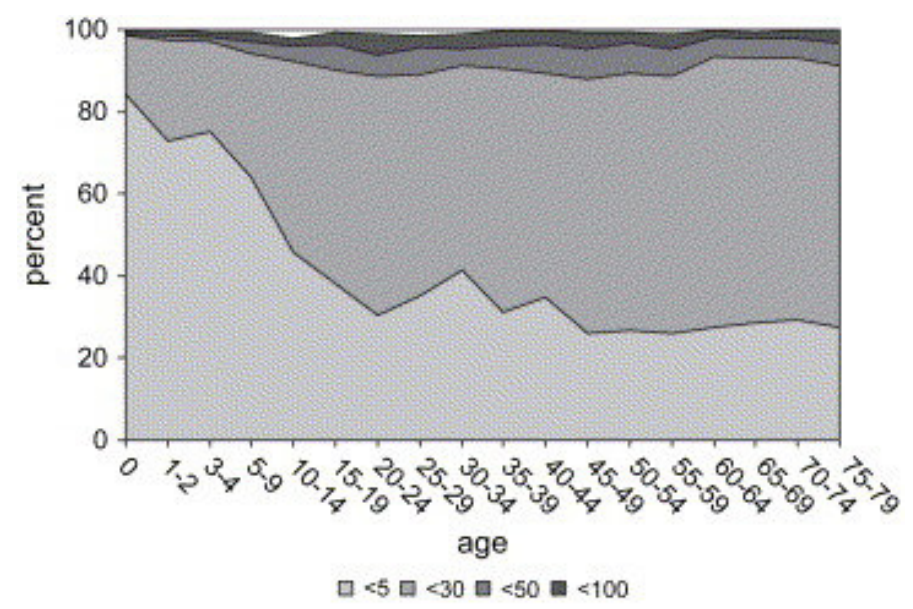

Figure 2. The age-specific IgG antibody levels in the population-based crosssectional study in the Netherlands. Note that the categories are cumulative, i.e. individuals in the lower categories also belong to all higher categories.

A series of cut-off values was chosen $(10,20,30,40,50,60,80,100$, and $150 \mathrm{U} / \mathrm{ml}$ ) for which we calculated the (age-specific) incidence of infection with $B$. pertussis in the population. The estimated numbers of infected individuals were corrected for the discrepancy between the age-distribution of the cross-sectional study population and the age-distribution of the Dutch population in 1996 by weighting each 1-year age-class with an appropriate correction factor. The estimates of incidence of infection were limited to 3-79-year olds. We did not 
estimate the incidence of infection in younger age-classes because IgG-PT in infants can be maternally derived and can be induced by vaccination (at the time of the study given at 3, 4, 5 and 11 months of age). Above 3 years of age, those factors do not influence the IgG-PT, because maternally derived antibodies disappear within 1 year, and IgG-PT induced by vaccination declines rapidly to very low or undetectable levels within 1 year after the last immunization. ${ }^{26}$ and 27

The average numbers of notified cases per 100,000 by age group in the period 1994-1996 were calculated using the age-distribution of the Dutch population in 1994-1996. The period of 1994-1996 was chosen for reasons of comparability with the period for which the number of infections was estimated. For each ageclass, the case-to-infection ratio could then be estimated by dividing the average numbers (per 100,000 ) of notified cases by the age-specific estimates of incidence of infection.

To assess the influence of individual variation in responses on the uncertainty of the incidence estimates we chose to compute a confidence interval for every cutoff value based on the following assumptions: (a) the longitudinal study population and the cross-sectional study population are the same in their responses to pertussis infection; (b) the variability in times since infection for different cut-off values is the same for the longitudinal and the cross-sectional study population; and (c) the log times since infection are normally distributed with mean and standard deviation calculated from the log times since infection from the longitudinal study.

\section{Results}

We describe the results for some of the cut-off values, namely the values 40,50 , 80 , and $100 \mathrm{U} / \mathrm{ml}$, because those values are considered to be the most conclusive for identifying recently infected individuals. After infection with $B$. pertussis the average time needed for the high IgG-PT level induced by infection to decline to $100 \mathrm{U} / \mathrm{ml}$ is 58.6 (CI [54.2,63.2]) days, to $80 \mathrm{U} / \mathrm{ml}$ it is 102.6 (CI [95.8, 109.8]) days, to $50 \mathrm{U} / \mathrm{ml}$ it is 208.9 (CI [195.4, 223.3]) days, and to $40 \mathrm{U} / \mathrm{ml}$ it is 297.6 (CI $[279.2,317.2]$ ) days (compare Fig. 1). Using a cut-off level of $100 \mathrm{U} / \mathrm{ml}$ the estimated percentage of the population that has been infected in the past 58.6 days was $0.84 \%$ (i.e. $0.84 \%$ of the population sera contained IgG-PT of at least $100 \mathrm{U} / \mathrm{ml}$ ) resulting in an estimated incidence in the year before serum sampling of $5.2 \%$ (365.25/58.6 times $0.84 \%)$. A cut-off value of $80 \mathrm{U} / \mathrm{ml}$ yielded an estimated incidence of $6.6 \%$ (365.25/102.6 times $1.86 \%)$, a cut-off value of $50 \mathrm{U} / \mathrm{ml}$ an estimated incidence of $6.6 \%$, and a cut-off value of $40 \mathrm{U} / \mathrm{ml}$ an estimated incidence of $7.2 \%$ in the year before serum sampling.

In summary, after standardization of the estimated incidences for various cut-off values to the time period of 1 year, one obtains remarkably consistent incidence estimates for a wide range of cut-off values (Fig. 3). 


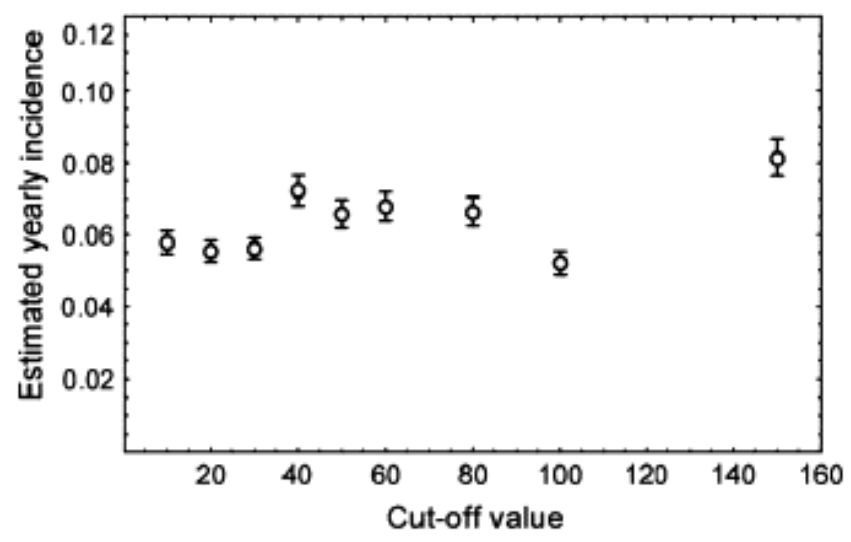

Figure 3. The estimated yearly incidence (as a fraction of the population) for different cut-off values and confidence intervals. It is questionable whether the estimates for cut-off values lower than $50 \mathrm{U} / \mathrm{ml}$ can be interpreted as valid estimates for recent incidence, but it is striking that even for those uncertain parameter regions estimates lie in the same range as for higher cut-off values.

The above results also hold for an age-stratified analysis, where the incidence of infection is estimated separately for each age category using the same range of cut-off levels of antibody titers. The consistency of the estimates for the yearly incidence of infection across different cut-off values indicates that the functional relationship between time since last infection and antibody titer as inferred from the longitudinal study $\mathrm{in}^{23}$ is a useful description of the immune response to IgGPT. In Fig. 4 the estimated age-specific incidence of infection with B. pertussis in the population is shown as calculated for the cut-off of $50 \mathrm{U} / \mathrm{ml}$. The estimated incidence of infection the year before blood sampling (1995-1996) amounted to 6571 per $100,000(6.6 \%)$ on average for 3-79-year olds. This was 685 times higher than the incidence of notified cases in the period 1994-1996 of 9.6 per $100,000(0.01 \%)$ for $3-79$-year olds (10.9 per 100,000 for all age groups), meaning that only one of 685 cases of infection is reported.

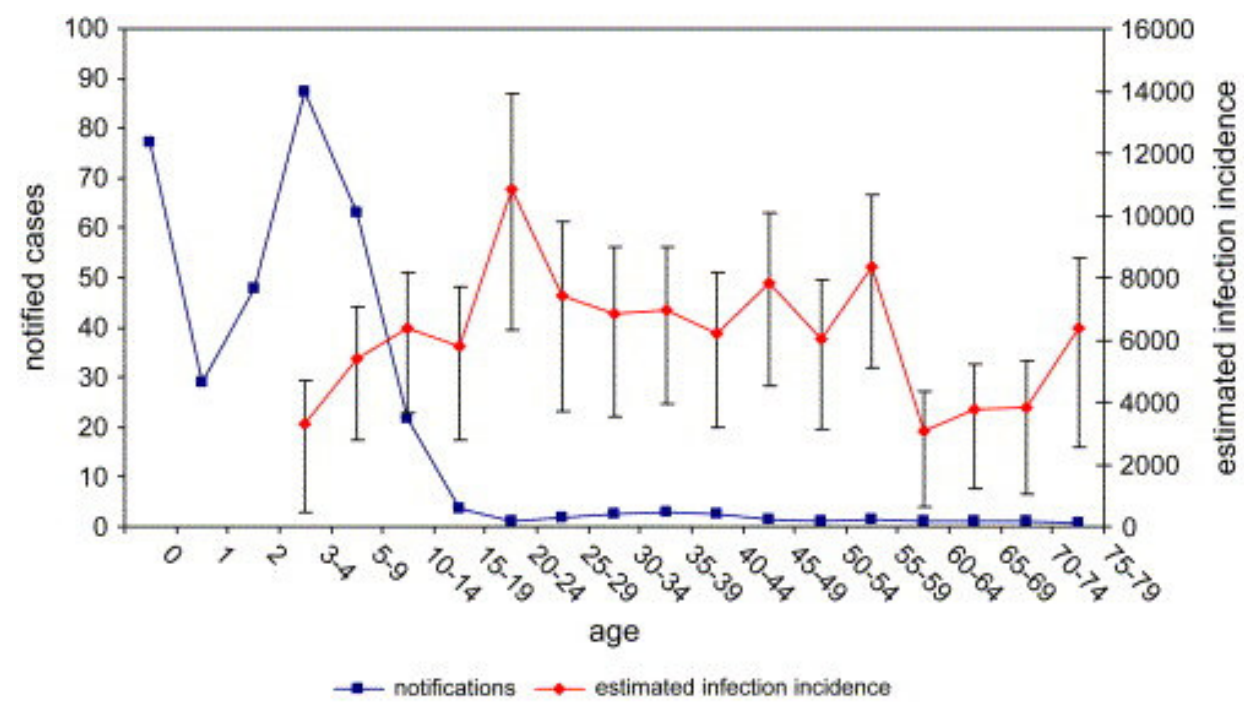

Figure 4. Estimated incidence of infection with $B$. pertussis per age group per 100,000 per year (open circles, right scale) and the annual incidence of notified cases of pertussis per age group per 100,000 averaged over the period 19941996 (filled circles, left scale). 
As shown in Fig. 4, the estimated incidence of infection is considerably higher for all age groups in comparison to the incidence of notified cases (note the different scales). There is a remarkable difference in the age-distributions of estimated infection rates vs. notified cases. The incidence of notified cases is high among 0year olds (77.2 per 100,000), 3-4 (87.4 per 100,000) and 5-9-year olds (63.1 per 100,000$)$ and decreases sharply till $20-24$ years $(1.2$ per 100,000$)$. For $30-$ 44-year olds a somewhat higher reported incidence is observed (2.6-2.8 per $100,000)$. For those aged between 45 and 69 years the incidences fluctuate between 1.2 and 1.6 per 100,000 , while the incidence is lowest for the oldest age groups (70 years and above). In contrast, the estimated incidence of infection is lowest for 3-4-year olds (3299 per 100,000). It increases sharply up to the age of 20-24 years $(10,831$ per 100,000$)$ and decreases again afterwards to a level of around 6500 per 100,000 in the age groups $25-55$ years. In the oldest age groups a further decrease to around 4000 per 100,000 can be seen.

\section{Discussion}

\section{Incidence and age profile of infections}

There is agreement in literature about the fact that pertussis vaccination reduces transmission. 28,29 and 30 To estimate the amount of that reduction requires knowledge about the fraction by age of clinical cases among all infections. This fraction is determined by a complex interplay of the age-dependent force of infection, immunity, and reporting behavior. Techniques of the analysis of serologic data have made it possible to identify individuals with recent $B$. pertussis infections and compare seroprevalence data with notifications of symptomatic disease. ${ }^{31,32 \text { and } 33}$

IgG-PT levels in the Netherlands can be interpreted as markers of recent infection, because firstly the amount of pertussis toxin in the Dutch whole cell vaccine is very low, and second, because vaccine-induced IgG-PT levels are minimal and short-lived. ${ }^{10}$ However, also in populations in which pertussis vaccines are used that contain moderate to high amounts of pertussis toxin, vaccine-induced IgG-PT declines to barely detectable levels within $2-4$ years. ${ }^{34,35 \text {, }}$ 36 and 37

Two major issues clearly emerge from our analysis. Firstly, the estimated incidence of infections with $B$. pertussis is considerable in all age groups and much higher than the reported incidence. ${ }^{1}$ We estimated that around $6.6 \%$ of the Dutch population had experienced infection with $B$. pertussis in the year before serum sampling, while, in contrast, the incidence of notifications in 1994-1996 amounted to $0.01 \%$ per year. ${ }^{1}$ Secondly, the age-specific profile of the reported cases diverges remarkably from the estimated age-specific profile of incidence of infection with $B$. pertussis in the population. While the highest incidence of reported symptomatic cases is observed among children aged 3-9 years, the incidence of infection is lowest among 3-4-year olds, increases with age and peaks for 20-24-year olds. Therefore, most cases are notified in those agecategories with the lowest incidence of infection.

Our cross-sectional study was performed in a limited time period (1995-1996). However, we believe that the age-specific profile of incidence of infection with $B$. pertussis is rather stable over time. Repeating the cross-sectional study can only assess whether or not this is correct. However, some support for our hypothesis is found in the similarity of the sero-profile of IgG-antibodies against pertussis toxin in 548 vaccinated children of $1-12$ years in 1980, the sero-profile of about 800 individuals of all ages in 1994 and the sero-profile in the present study in 
1995-1996. ${ }^{38}$ Also, the fact that our findings in adolescents and adults are in agreement with the high incidences (3.3-8\% per year) found in prospectively followed cohorts of small numbers of adolescents and adults in the USA in other time periods may be seen as supporting our hypothesis. ${ }^{6,39}$ and 40 The immune response of IgG-antibodies against pertussis toxin after infection with $B$. pertussis shows large variation among individuals. ${ }^{23}$ Here, we worked with the point wise (for each titer value) averages of the individual response curves (Fig. 1). We applied this procedure to a large representative population sample, and therefore we assumed that variation on the individual level averages out on the population level. We assumed that the longitudinal study population and the cross-sectional study population are identical in their responses to pertussis infection. With the size of the longitudinal sample as it is available to us at present, we can only say that the responses in different patient categories did appear to be similar. ${ }^{23}$ In a more recent study which also included patients of older age groups it was shown that age did not significantly affect the rise, peak and decline of IgG-PT antibody titres after infection. ${ }^{41}$

Several factors are responsible for the large discrepancies between reported pertussis cases and the estimated cases of infection in their incidences and ageprofiles. The amount of underreporting varies by age, because severity of disease, medical care seeking and diagnostic power are varying with age. Indeed, a high rate of underreporting has been observed mainly in older children,

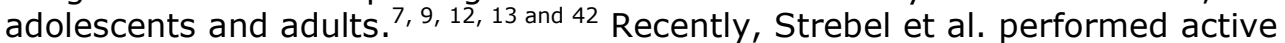
case finding among older children and adults (10-49 years). ${ }^{43}$ An incidence of symptomatic infection with $B$. pertussis of $0.5 \%$ per year was found, which was about 100 -fold higher than the incidence of notified cases in that age category. A similar high incidence of symptomatic infections with $B$. pertussis among adults was found in a highly vaccinated region of France. ${ }^{44}$

Another set of factors that influence the age-specific incidence profile of pertussis infections is related to the dynamics of transmission and immunity. The transmission of air-borne infections is strongly determined by the age-dependent patterns of mixing in a population ${ }^{45}$ The peaks observed in Fig. 4 of age-specific incidence in the population might be related to high contact rates, the lower infection rate for those aged 60 years and older to a lower contact rate in that age group. The gradual increase with age of the incidence of infection to a peak of $10.8 \%$ in $20-24$-year olds suggests that there is a high variability in the duration of vaccine-induced immunity, which in some may be less than 2 years, in others more than 10 years. However, the incidence of notified cases of pertussis is highest among 3-9-year olds, suggesting a strong reporting bias in that age category, but also showing that susceptibility for symptomatic infection with $B$. pertussis in some may re-emerge shortly after vaccination. The mean incidence of $6.6 \%$, if constant in time, indicates that on average, within a period of 20 years the entire population experiences infection, i.e. that vaccination against pertussis will be followed on average by three episodes of natural infection during life. Indeed, there are strong indications that immunity wanes also after natural infection and that re-infection is possible. German investigators estimated the duration of the protective period following natural infection at 20 years. ${ }^{46}$ Versteegh et al. recently described four individuals in whom re-infection was documented, respectively, 3.5, 5, 6 and 12 years after the first. ${ }^{25}$

\section{Implications for vaccination}

In the Netherlands, the age-specific profile of notifications with the highest incidence in those aged 3-9 years led to the decision of the Dutch Health Council to introduce a booster vaccination with acellular pertussis vaccine at 4 years of 
age. ${ }^{47}$ Since siblings play a role in transmission to vulnerable infants, ${ }^{14 a n d}{ }^{15}$ one expects the incidence of severe pertussis in infants to decline. However, on the basis of the incidence estimates presented here, we expect that the introduction of the booster vaccination at 4 years on the long run could postpone infection to older age groups. Since these age groups may have more contacts with vulnerable infants, this could imply that on the long run booster vaccination might even lead to an increase of the incidence of severe pertussis in infants.

\section{Conclusion}

More insight is needed into the role of adults as compared to siblings in the transmission of $B$. pertussis to young unvaccinated infants. The results of the present study support the findings of others in that adults are an important source of infection. ${ }^{8}, 12,13,14$ and 16 Mathematical modeling studies are needed to study the effects of different vaccination strategies on the age-specific incidence of (symptomatic and asymptomatic) pertussis infections. Previous modeling studies 48 and 49 had to cope with the lack of data concerning the force of infection and the age-dependent fractions of symptomatic and notified cases of infection. While we are still far from having a solid quantitative basis on which to build reliable mathematical models, we think that our study investigated an essential link between the transmission dynamics as described by mathematical models and notification data of pertussis infections. This methodology can be used more generally to estimate infection frequency from seroprevalence data.

\section{References}

1 H.E. de Melker, J.F.P. Schellekens, S.E. Neppelenbroek, F.R. Mooi, H.C. Rümke and M.A.E. Conynvan Spaendonck, Reemergence of pertussis in the highly vaccinated population of the Netherlands: observations on surveillance data, Emerg Infect Dis 6 (2000), pp. 348-357.

2 G. De Serres, N. Boulianne, M. Douville Fradet and B. Duval, Pertussis in Quebec: ongoing epidemic since the late 1980s, Can Commun Dis Rep 21 (1995), pp. 45-48.

3 R. Andrews, A. Herceg and C. Roberts, Pertussis notifications in Australia, 1991 to 1997, Commun Dis Intell 21 (1997), pp. 145-148.

4 D. Güris, P.M. Strebel and B. Bardenheier, Changing epidemiology of pertussis in the United States: increasing reported incidence among adolescents and adults, 1990-1996, Clin Infect Dis 28 (1999), pp. $1230-1237$.

5 L.D. Senzilet, S.A. Halperin, J.S. Spika, M. Alagaratnam, A. Morris and B. Smith et al., Pertussis is a frequent cause of prolonged cough illness in adults and adolescents, Clin Infect Dis 32 (2001), pp. 1691-1697.

6 B.A. Cromer, J. Goydos, J. Hackell, J. Mezzatesta, C. Dekker and E.A. Mortimer, Unrecognized pertussis infection in adolescents, Am J Dis Child 147 (1993), pp. 575-577.

7 W.K. Yih, S.M. Lett, F.N. des Vignes, K.M. Garrison, P.L. Sipe and C.D. Marchant, The increasing incidence of pertussis in Massachusetts adolescents and adults, 1989-1998, J Infect Dis 182 (2000), pp. $1409-1416$

8 J.D. Cherry, The role of Bordetella pertussis infections in adults in the epidemiology of pertussis, Dev Biol Stand 89 (1997), pp. 181-186.

9 G. Serres de, R. Shadmani, B. Duval, N. Boullianne, P. Déry and M. Douville Fradet et al., Morbidity of pertussis in adolescents and adults, J Infect Dis 182 (2000), pp. 174-179.

10 H.E. de Melker, F.G.A. Versteegh, M.A.E. Conyn-van Spaendonck, L.H. Elvers, G.A.M. Berbers and A. van der Zee et al., Specificity and sensitivity of high levels of immunoglobulin $G$ antibodies against pertussis toxin in a single serum sample for diagnosis of infection with Bordetella pertussis, J Clin Microbiol 38 (2000), pp. 800-806.

$11 \mathrm{P}$. Gardner, Indications for acellular pertussis vaccines in adults: the case for selective rather than universal recommendations, Clin Infect Dis 28 (1999), pp. S131-S135.

12 J.D. Nelson, The changing epidemiology of pertussis in young infants: the role of adults as a reservoir of infection, Am J Dis Child 132 (1978), pp. 371-373. 
13 J.L. Deen, C.A.M. Mink, J.D. Cherry, P.D. Christenson and E.F. Pineda et al., Household contact study of Bordetella pertussis infections, Clin Infect Dis 21 (1995), pp. 1211-1219.

14 S. Baron, E. Njamkepo, E. Grimprel, P. Begue, J. Desenclos and J. Drucker et al., Epidemiology of pertussis in French hospitals in 1993 and 1994: thirty years after a routine use of vaccination, Pediatr Infect Dis J 17 (1998), pp. 412-418.

15 I. Srugo, D. Benilevi, R. Madeb, S. Shapiro, T. Shohat and E. Somekh et al., Pertussis infection in fully vaccinated children in day-care centers, Israel, Emerg Infect Dis 6 (2000), pp. 526-529.

16 E. Grimprel, S. Baron, D. Lévy-Bruhl, J.M. Garnier, N'jamkepo and N. Guiso et al., Influence of vaccination coverage on pertussis transmission in France, Lancet 354 (1999), pp. 1699-1700.

17 S.S. Long, C.J. Welkon and J.L. Clark, Widespread silent transmission of pertussis in families: antibody correlates of infection and symptomatology, J Infect Dis 161 (1990), pp. 480-486.

18 E. Elliot, P. McIntyre, G. Ridley, A. Morris, J. Massie and J. McEniery et al., National study of infants hospitalized with pertussis in the acellular vaccine era, Pediatr Infect Dis 23 (2004), pp. 246-252.

19 H.S. Izurieta, T.A. Kenyon, P.M. Strebel, A.L. Baughman, S.T. Shulman and M. Wharton, Risk factors for pertussis in young infants during an outbreak in Chicago in 1993, Clin Infect Dis 22 (1996), pp. 503-507.

20 K. Edwards, Pertussis: an important target for maternal immunization, Vaccine 21 (2003), pp. 3483-3486.

21 F.M. Müller, J.E. Hoppe and C.H. Wirsing-von König, Laboratory diagnosis of pertussis: state of the art in 1997, J Clin Microbiol 35 (1997), pp. 2435-2443.

22 S.A. Halperin, R. Bortolussi, A. Kasina and A.J. Wort, Use of a Chinese hamster ovary cell cytotoxicity assay for the rapid diagnosis of pertussis, J Clin Microbiol 28 (1990), pp. 32-38.

23 P.F.M. Teunis, O.G. van der Heijden, H.E. de Melker, J.F.P. Schellekens, F.G.A. Versteegh and M. Kretzschmar, Kinetics of IgG-antibody response to pertussis toxin after infection with B. pertussis, Epidemiol Infect 129 (2002), pp. 479-489.

24 H.E. de Melker and M.A.E. Conyn-van Spaendonck, Immunosurveillance and the evaluation of national immunisation programmes: a population-based approach, Epidemiol Infect 121 (1998), pp. 637-643.

25 F.G.A. Versteegh, J.F.P. Schellekens, A.F. Nagelkerke and J.J. Roord, Laboratory confirmed reinfection with Bordetella pertussis, Acta Paediatr 91 (2002), pp. 95-97.

26 J. Nagel, S. de Graaf and D. Schijf-Evers, Improved serodiagnosis of whooping cough caused by Bordetella pertussis by determination of IgG anti-LFP antibody levels, Dev Biol Stand 61 (1985), pp. 325-330.

27 J. Labadie, L.C. Sundermann, H.C. Rümke and The DTP-IPV-Hib Vaccine Study Group, Multi-center study on the simultaneous administration of DTP-IPV and Hib PRP-T vaccines. Part 1. Immunogenicity, National Institute of Public Health and the Environment, Bilthoven, The Netherlands (1996) Report No. 124001003.

28 P.E.M. Fine and J.A. Clarkson, The recurrence of whooping cough: possible implications for assessments of vaccine efficacy, Lancet 1 (1982), pp. 666-669.

29 P. Rohani, D.J.D. Earn and B.T. Grenfell, Impact of immunisation on pertussis transmission in England and Wales, Lancet 355 (2000), pp. 285-286.

30 N.J. Gay and E. Miller, Pertussis transmission in England and Wales, Lancet 355 (2000), pp. 15531554

31 C.M. Mink, J.D. Cherry, P.D. Christenson, K. Lewis, E. Pineda and D. Shlian et al., A search for Bordetella pertussis infection in university students, Clin Infect Dis 14 (1992), pp. 464-471.

32 A. Nardone, R.G. Pebody, P.A.C. Maple, N. Andrews, N.J. Gay and E. Miller, Seroepidemiology of Bordetella pertussis in England and Wales, Vaccine 22 (2004), pp. 1314-1319.

33 R.G. Pebody, N.J. Gay, A. Giammanco, S. Baron, J. Schellekens and A. Tischer et al., The seroepidemiology of Bordetella pertussis infection in Western Europe, Epidemiol Infect 133 (2005), pp. 159-171.

34 J.D. Cherry, T. Beer, S.A. Chartrand, J. DeVille, E. Beer and M.A. Olsen et al., Comparison of values of antibody to Bordetella pertussis antigens in young German and American men, Clin Infect Dis 20 (1995), pp. 1271-1274.

35 J. Storsaeter, H.O. Hallander, L. Gustafsson and P. Olin, Levels of anti-pertussis antibodies related to protection after household exposure to Bordetella pertussis, Vaccine 16 (1998), pp. 1907-1916.

36 S.A. Halperin, D. Scheifele, L. Barreto, C. Pim, R. Guasparini and L. Medd et al., Comparison of a fifth dose of a five-component acellular or a whole cell pertussis vaccine in children four to six years of age, Pediatr Infect Dis J 18 (1999), pp. 772-779. 
37 M.E. Pichichero, K.M. Edwards, E.L. Anderson, M.B. Rennels, J.A. Englund and D.E. Yerg et al., Safety and immunogenicity of six acellular pertussis vaccines and one whole-cell pertussis vaccine given as a fifth dose in four- to six-year-old children, Pediatrics 105 (2000), p. e11.

38 H.E. de Melker, H.G.L. Boshuis, M.P.T.M. Mommers, L.C. Sundermann, J. Labadie and H.C. Rümke et al., Serodiagnostiek van kinkhoest: interpretatie van hoge IgG/IgA concentraties in acute fase serum: (Her)evaluatie van eenpuntsserologie (1996) RIVM-report No. 128507003, Bilthoven.

39 J.G. Deville, J.D. Cherry, P.D. Christenson, E. Pineda, C.T. Leach and T.L. Kuhls et al., Frequency of unrecognized Bordetella pertussis infections in adults, Clin Infect Dis 21 (1995), pp. 639-642.

40 S.L. Hodder, J.D. Cherry, E.A. Mortimer Jr., A.B. Ford, J. Gornbein and K. Papp, Antibody responses to Bordetella pertussis antigens and clinical correlations in elderly community residents, Clin Infect Dis 31 (2000), pp. 7-14.

41 F.G.A. Versteegh, P.L.J.M. Mertens, H.E. de Melker, J.J. Roord, J.F.P. Schellekens and P.F.M. Teunis, Age-specific long-term course of IgG antibodies to pertussis toxin after symptomatic infection with Bordetella pertussis, Epidemiol Infect 133 (2005), pp. 737-748.

42 W.A. Orenstein, Pertussis in adults: epidemiology, signs, symptoms, and implications for vaccination, Clin Infect Dis 28 (1999), pp. S147-S150.

43 P. Strebel, J. Nordin, K. Edwards, J. Hunt, J. Besser and S. Burns et al., Population-based incidence of pertussis among adolescents and adults, Minnesota, 1995-1996, J Infect Dis 183 (2001), pp. 1353-1359.

44 S. Gilberg, E. Njamkepo, I.P. du Chatelet, H. Partouche, P. Gueirard and C. Ghasarossian et al., Evidence of Bordetella pertussis infection in adults presenting with persistent cough in a French area with very high whole-cell vaccine coverage, J Infect Dis 186 (2002), pp. 415-418.

$45 \mathrm{~J}$. Wallinga, W.J. Edmunds and M. Kretzschmar, Human contact patterns and the spread of airborne infectious diseases, Trends Microbiol 7 (1999), pp. 372-377.

46 C.H. Wirsing von Konig, S. Postels-Multani, H.L. Bock and H.J. Schmitt, Pertussis in adults: frequency of transmission after household exposure, Lancet 346 (1995), pp. 1326-1329 Erratum in Lancet 346 (1995), p. 1502.

47 G.D. van Dijk, Invoering extra kinkhoest-vaccinatie, Infect Bull 11 (2000), pp. 177-178.

48 M. van Boven, H.E. de Melker, J.P.F. Schellekens and M. Kretzschmar, A model based evaluation of the 1996-7 pertussis epidemic in the Netherlands, Epidemiol Infect 127 (2001), pp. 73-85.

49 A. van Rie and H.W. Hethcote, Adolescent and adult pertussis vaccination: computer simulations of five new strategies, Vaccine 22 (2004), pp. 3154-3165. 